\title{
Putative Intermediates in the Nerve Cell Differentiation Pathway in Hydra Have Properties of Multipotent Stem Cells
}

\author{
Thomas W. Holstein and Charles N. David \\ Department of Zoology, University of Munich, Munich, Germany
}

Accepted August 6, 1990

\begin{abstract}
We have investigated the properties of nerve cell precursors in hydra by analyzing the differentiation and proliferation capacity of interstitial cells in the peduncle of Hydra oligactis, which is a region of active nerve cell differentiation. Our results indicate that about $50 \%$ of the interstitial cells in the peduncle can grow rapidly and also give rise to nematocyte precursors when transplanted into a gastric environment. If these cells were committed nerve cell precursors, one would not expect them to differentiate into nematocytes nor to proliferate apparently without limit. Therefore we conclude that cycling interstitial cells in peduncles are not intermediates in the nerve cell differentiation pathway but are stem cells. The remaining interstitial cells in the peduncle are in G1 and have the properties of committed nerve cell precursors (Holstein and David, 1986). Thus, the interstitial cell population in the peduncle contains both stem cells and noncycling nerve precursors. The presence of stem cells in this region makes it likely that these cells are the immediate targets of signals which give rise to nerve cells. 1990 Academic Press, Inc.
\end{abstract}

\section{INTRODUCTION}

Multipotent interstitial stem cells in hydra continously differentiate nerve cells, nematocytes and gland cells (David and Gierer, 1974; Bode and David, 1978; David et al., 1987). Nerve cell differentiation occurs primarily in head and foot regions of hydra and is stimulated by head and foot regeneration or by treatment with a neuropeptide, the head activator (Schaller, 1976; Holstein et al., 1986). The nature of the target cell which differentiates nerve cells in response to regeneration signals or HA treatment is presently not known. It could be a multipotent stem cell or an already committed, cycling nerve precursor.

Recently Heimfeld and Bode (1984) and Bode et al. (1990) have suggested that the nerve cell differentiation pathway contains cycling intermediates. Evidence for this hypothesis was based on the observation that some cells having the morphology of typical nerve precursors (Heimfeld and Bode, 1984; Holstein and David, 1986) could be labeled with $\left[{ }^{3} \mathrm{H}\right]$ thymidine.

To investigate this hypothesis in more detail we have analyzed the properties of interstitial cells present in tissue which displays extensive nerve cell differentiation. The peduncle of the stalked hydra Hydra oligactis is well suited for such experiments since it is a site of active nerve cell differentiation (Bode et al., 1990) and since it contains higher numbers of interstitial cells, which are putative nerve cell precursors, than other species. In the present experiments we have determined the differentiation and proliferation capacity of interstitial cells in peduncles of Hydra oligactis both in situ and by explanting them from the peduncle into a gastric environment which can support stem cell proliferation.

The results indicate that most interstitial cells in the peduncle are postmitotic G1 cells similar to nerve precursors which have been described previously (Holstein and David, 1986). A fraction of the peduncle cells, however, have S and G2 nuclear DNA contents and can be labeled with $\left[{ }^{3} \mathrm{H}\right]$ thymidine. When transplanted to a gastric environment, these cells grow rapidly and reconstitute an interstitial cell population typical of the gastric region. In particular, these proliferating cells give rise to intermediates in the nematocyte differentiation pathway. Thus, they exhibit the properties of multipotent stem cells in hydra. Since in a region of active nerve cell differentiation only interstitial cells with the properties of stem cells or postmitotic nerve cell precursors were present, our data do not support the idea of cycling nerve precursors. Rather, they suggest that commitment to nerve cell differentiation occurs directly at the level of stem cells.

\section{MATERIALS AND METHODS}

Animal culture. All experiments were carried out using a male strain of $H$. oligactis originally collected from Lake Zürich in Switzerland in 1979 by Dr. Lynne Littlefield and kindly provided by Dr. Pierre Tardent 1983. The animals were cultured in M-solution, adjusted to pH 7.8 (Sugiyama and Fujisawa, 1977). Budless hydra were used in all experiments. Animals were selected from the culture $22 \mathrm{hr}$ after the last feeding.

Identification of cell types by maceration. The cell 
TABLE 1

Cell Composition of the Lower Peduncle of Hydra oligactis

Number of cells/ proximal peduncle

Epithelial cells

Interstitial cells, total

Small interstitial cells

Large interstitial cells

Nests of four interstitial cells (4s)
Nerve cells

$1400 \pm 360$
$346 \pm 34$
$57 \pm 10$
$44 \pm 11$
$13 \pm 4$
$1 \pm 1$

composition of hydra tissue was determined in macerated preparations (David, 1973) as described previously (Holstein and David, 1990).

Determination of cell size. The cell size of interstitial cells and their nuclei was analyzed by measuring the area of cells in macerated preparations as described by Holstein and David (1990). Since the cells are effectively flat under the coverslip (thinner than $4 \mu \mathrm{m}$ ), the area of a given cell is a good approximation of its volume.

Determination of nuclear DNA content. The fluorescence of nuclei of interstitial cells was measured with a Leitz Dialux microscope fitted with a Leitz epifluorescence attachment using filter block $\mathrm{A}(365 / 450 \mathrm{~nm})$ as described by Holstein and David (1990).

Labeling with $\left[{ }^{3} H\right]$ thymidine and autoradiography. Hydra were labeled with $\left[\right.$ methyl $\left.{ }^{3} \mathrm{H}\right]$ thymidine $(44 \mathrm{Ci} /$ mmole, Amersham) by injecting the isotope into the gastric cavity using a polyethylene needle (David and Campbell, 1972) as described (Holstein and David, 1990).

Grafting procedures. Tissue grafting was done according to procedure of Rubin and Bode (1978). Because of the very small diameter of peduncle and head pieces, nylon fish line which fits inside $\mathrm{PE}-10$ polyethylenetubing (Clay-Adams, Becton-Dickinson) was used for grafting.

Nitrogen mustard treatment. Animals were treated with $0.01 \%$ nitrogen mustard (NM) as described by Diehl and Burnett (1964) and fed once thereafter. Tissue from treated animals was free of interstitial cells after 6 days.

\section{RESULTS AND DISCUSSION}

\section{Cellular Composition of the Peduncle}

The peduncle in hydra is defined as the region between the lowest bud and the basal disc. The cellular composition of the peduncle is different from the gastric tissue: it has a high concentration of nerve cells, reduced numbers of interstitial cells, and no differentiating nematocytes (Bode et al., 1973). Many of the interstitial cells in this tissue exhibit the morphology of nerve cell precursors (Bode et al, 1990).

In the experiments described here, we have used the stalked hydra $H$. oligactis as a source of peduncle tissue because in this species the peduncle is larger than in other species. To exclude any possible contribution of gastric cells to the experimental observations, we have only used the proximal half of the peduncle. Table 1 shows the cellular composition of this tissue. There are large numbers of nerve cells and small interstitial cells, but very few large interstitial cells and practically no precursors in the nematocyte differentiation pathway (4s). The total number of interstitial cells in these lower peduncles varied from 50 to 500 depending on the condition of the hydra culture. Such fluctuations in the animals have also been reported by Bode et al. (1990). All
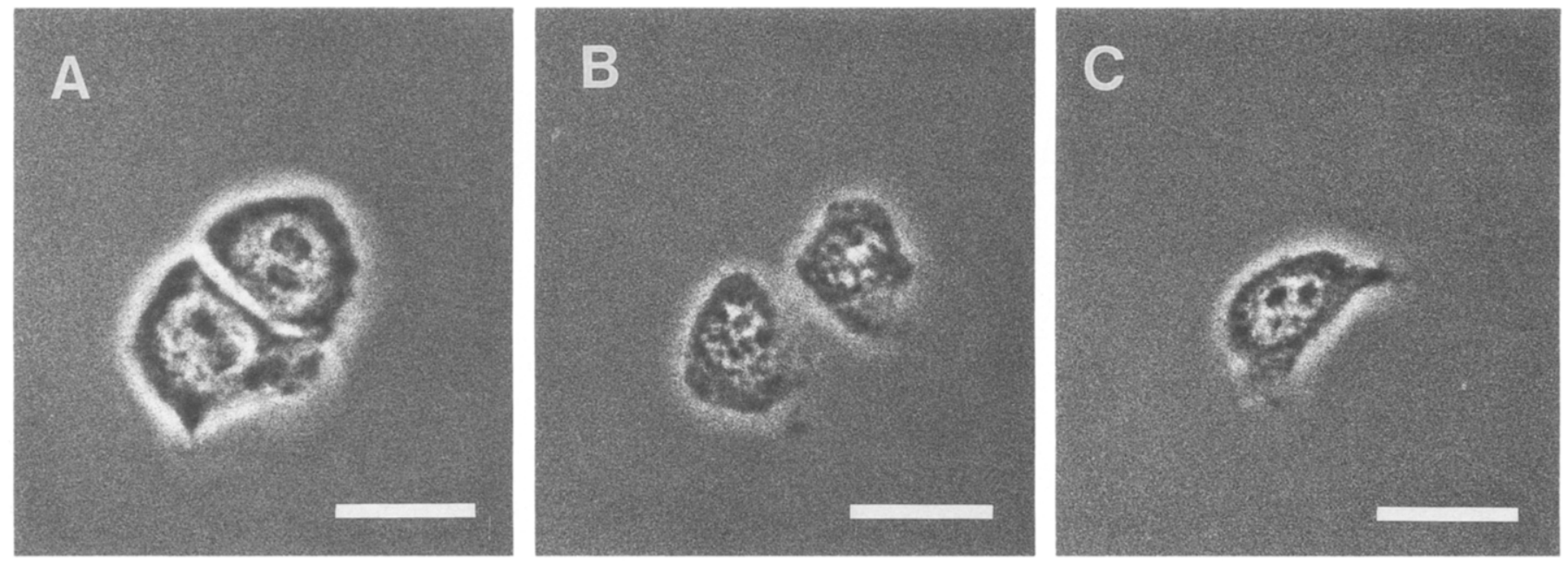

FIG. 1. Size and morphology of interstitial cells in the peduncle. (A) large interstitial cells. (B) and (C) small interstitial cells. Micrographs are from macerated cell preparations, phase contrast, bar indicates $10 \mu \mathrm{m}$. 


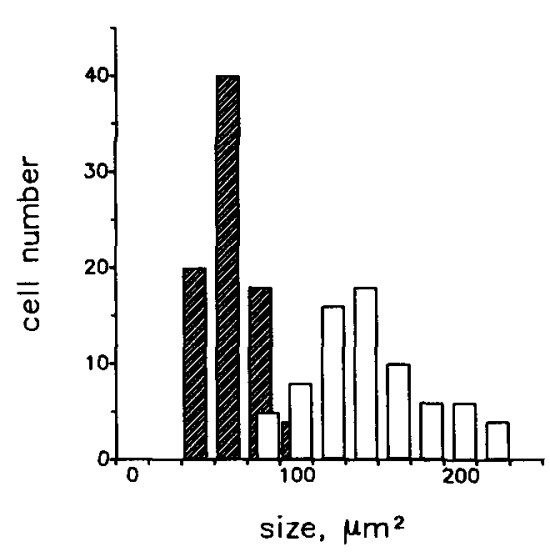

Frg. 2. Size distribution of large and small interstitial cells in the peduncle. Shaded bars: small interstitial cells. Open bars: large interstitial cells. The measurements of large and small interstitial cells do not reflect their relative proportions in tissue.

the experiments reported here involved animals with cell numbers as shown in Table 1.

\section{Size and Morphology of Interstitial Cells in Peduncles}

Figure 1 shows examples of the morphology of interstitial cells in peduncle tissue. Both large interstitial cells having a nucleus with a prominent nucleolus (Fig. $1 \mathrm{~A})$ and small interstitial cells with condensed heterochromatic nuclei and no discernible nucleoli (Fig. 1B, $1 \mathrm{C})$ are present. Figure $2 \mathrm{~A}$ demonstrates that the visual impression of size differences between large and small interstitial cells can be confirmed by quantitative size measurements in macerates. Small interstitial cells in the proximal peduncle have an average area of $79 \mu \mathrm{m}^{2}$, while large interstitial cells in this tissue have an average size of $130 \mu \mathrm{m}^{2}$ (Fig. 2). Since small interstitial cells are about half the size of large interstitial cells, they could be mitotic products of these cells. Since their size and nuclear morphology are similar to nerve cells, these small interstitial cells are likely to be the direct precursors to nerve cells (Holstein and David, 1986; Bode et al. 1990).

\section{Cell Cycle Parameters of Interstitial Cells in the Peduncle}

We have shown previously that nerve cell precursors induced by head activator treatment are arrested in G1 of the cell cycle (Holstein and David, 1986). Therefore we measured the DNA content of the interstitial cells in the peduncle of $H$. oligactis (Fig. 3A). About $60-70 \%$ of the interstitial cells in peduncles had a G1 nuclear DNA content typical of nerve cell precursors. The remaining cells had S/G2 nuclear DNA contents and thus appeared to be in cycle. Consistent with this finding, $18 \%$ of the
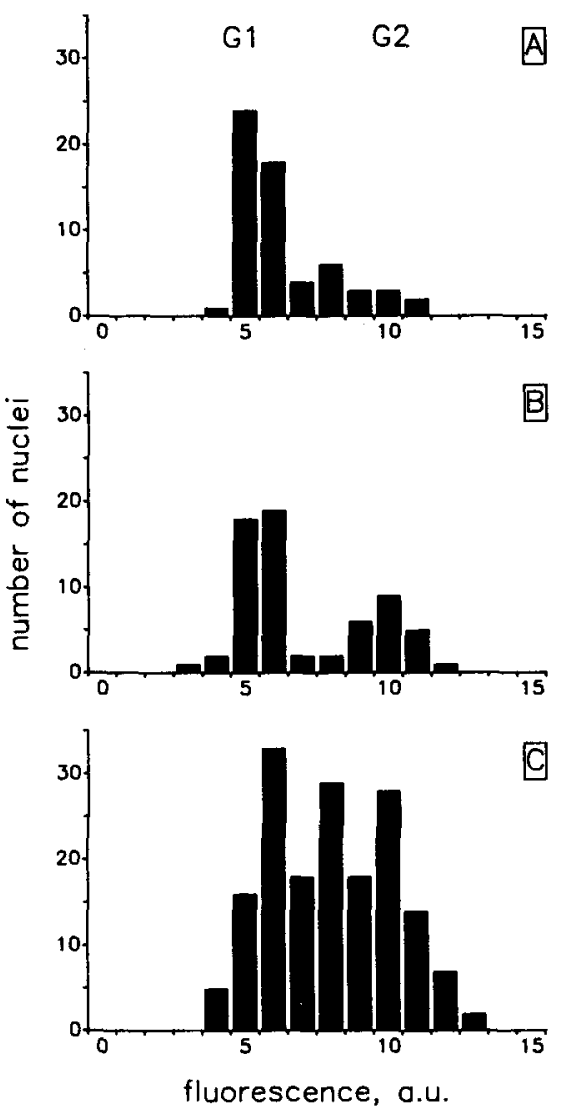

FIG. 3. Nuclear DNA content of total interstitial cells from the peduncle determined by microfluorometry. (A) 0 days, (B) 3 days, (C) 5 days after grafting to NM head. Nuclei of nerve cells (G1 DNA content, David and Gierer, 1974) were used as a reference to determine the positions of G1 and G2. Abscissa: DAPI fluorescence in arbitary units.

interstitial cells in the peduncle could be pulse labeled with $\left[{ }^{3} \mathrm{H}\right]$ thymidine (see also Fig. 6 ).

On the basis of their nuclear morphology, G1 nuclear DNA content and size, it appears that most small inter-

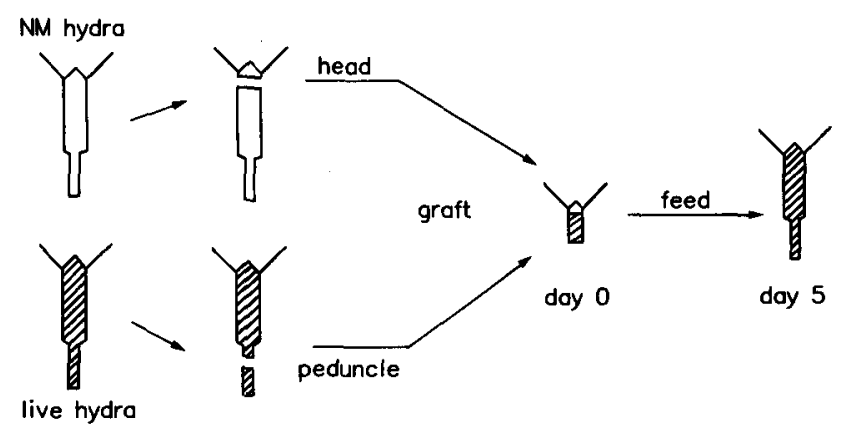

FIG. 4. Grafting procedure used to measure the growth and differentiation of peduncle interstitial cells transplanted to a gastric environment. Heads from 6-day nitrogen mustard (NM)-treated animals (free of interstitial cells) were grafted onto the proximal peduncle of normal hydra; the grafts were fed daily. 


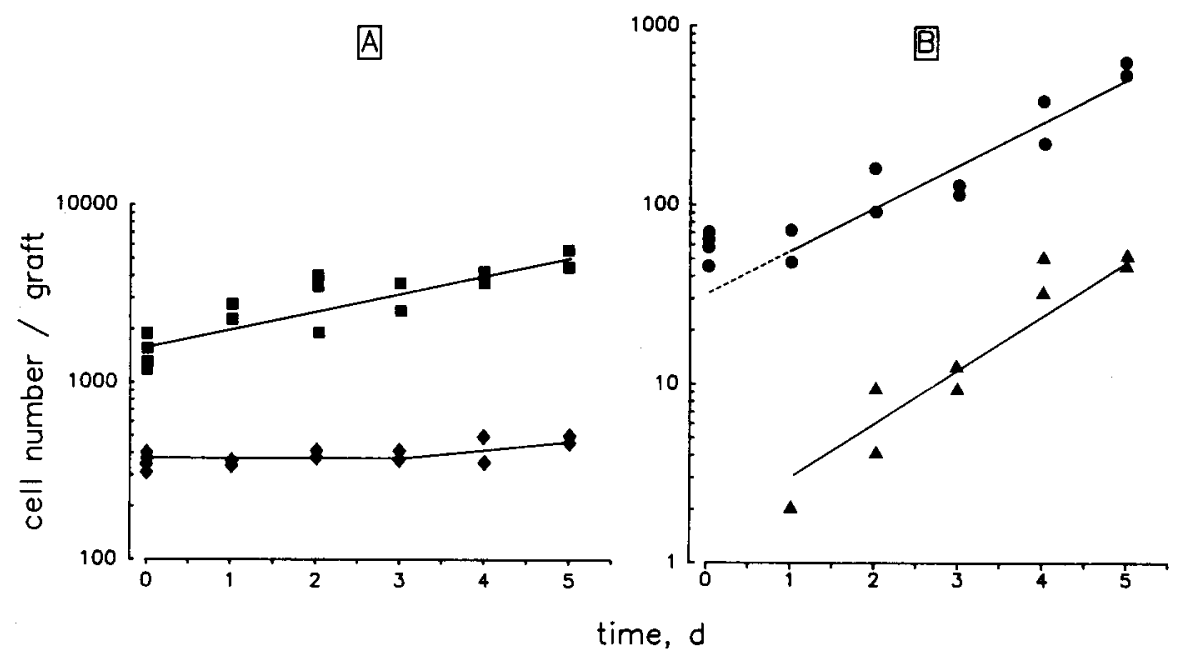

FIG. 5. Growth and differentiation of interstitial cells in peduncles grafted to NM heads. Epithelial cells $(\boldsymbol{\square})$ and nerve cells $(\bullet)$, total interstitial cells $(1 s+2 s)(\bullet)$ and nematocyte precursors $(4 s)(\Delta)$.

stitial cells in peduncles are nerve precursors similar to those described previously (Holstein and David, 1986). The function of peduncle interstitial cells which have S/G2 nuclear DNA contents and can be labeled with $\left[{ }^{3} \mathrm{H}\right]$ thymidine is unclear. These could be cycling stem cells or cycling intermediates in the nerve cell differentiation pathway (Heimfeld and Bode, 1984; Bode et al., 1990).

\section{Interstitial Cells from the Peduncle Proliferate in a Gastric Environment}

To test the differentiation and proliferation capacity of interstitial cells from peduncle tissue, we trans-

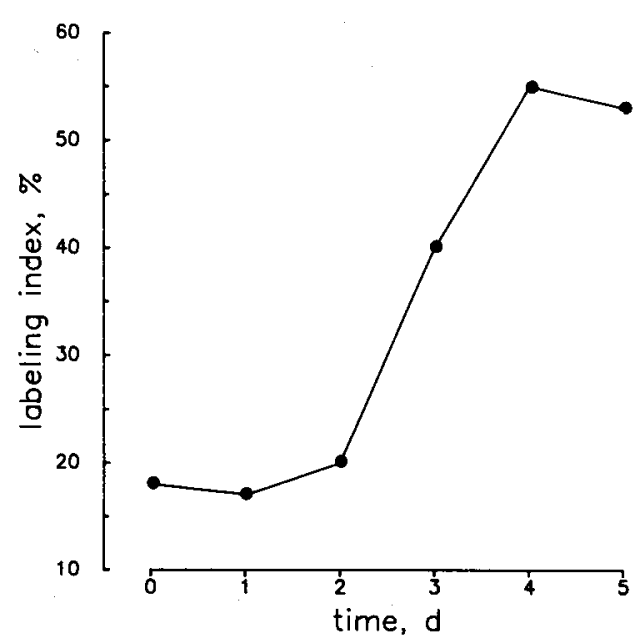

FIG. 6. $\left[{ }^{3} \mathrm{H}\right]$ Thymidine pulse labeling index of total interstitial cells growing in peduncles grafted to NM heads. Abscissa: days after grafting. planted them to a nonpeduncle environment, in this case the gastric region. If these cells are committed precursors to nerve differentiation, then they should differentiate into nerve cells following transplantation to a nonpeduncle environment. On the other hand, if they are stem cells, they should grow exponentially and reconstitute the normal interstitial cell composition of gastric tissue, including differentiating nematoblasts.

To transplant peduncle cells to a gastric environment, we grafted heads of nitrogen mustard-treated animals, which are devoid of interstitial cells, onto lower peduncles of normal animals and fed the grafts daily (Fig. 4). Such grafts regenerate to normal animals with a typical gastric region within several days. In such grafts the epithelial cells grew rapidly but the number of nerve cells did not change over the course of several days (Fig. $5 \mathrm{~A})$. As a result the ratio of nerve cells to epithelial cells decreased dramatically from 0.30 at Day 0 , which is typical of peduncle tissue, to 0.09 at Day 5 , which is typical of gastric tissue.

Interstitial cells present in the peduncle at the time of grafting grew rapidly in the regenerated gastric environment (Fig. 5B) beginning on Day 1. Regression analysis of the growth rate from $t_{1}$ to $t_{5}$ indicates that the cells had a doubling time of 1.2 days. Extrapolation of the growth curve to $t_{0}$ indicates that growth of the interstitial cell population started from about 30 cells at the time of grafting. Since the peduncle tissue contained about 60 total interstitial cells on Day 0 , this result suggests that about half of these peduncle cells were capable of extensive proliferation.

The rapid growth of interstitial cells in the grafted peduncles was accompanied by a shift in the distribution of nuclear DNA contents from primarily G1 cells on 
Day 0 to primarily S and G2 cells on Day 5 (Figs. 3A$3 \mathrm{C}$ ). Consistent with this result, the pulse-labeling index increased from 0.2 on Day 0 to 0.6 on Day 4 (Fig. 6). The relatively slow change in both the nuclear DNA distribution and in the labeling index is due to the persistence of noncycling G1 cells for several days after grafting (Fig. 3B).

Growth appears to occur from the S/G2 population since most G1 cells are postmitotic nerve precursors. S/G2 cells, however, constitute only about $30-40 \%$ of total peduncle interstitial cells (Fig. $3 \mathrm{~A}$ ) whereas growth occurs from roughly $50 \%$ of peduncle interstitial cells (Fig. 5). This discrepancy could be due to errors in the estimates. However, it appears more likely that some "G1" cells are not postmitotic nerve precursors but cycling cells in early S, i.e., part of the S/G2 population (Holstein and David, 1986).

\section{Interstitial Cells from the Peduncle Differentiate Nematocyte Precursors in a Gastric Environment}

The rapid exponential growth of interstitial cells in the grafted peduncles suggests that these cells are stem cells capable of regenerating the typical interstitial population of normal animals. If this is true, they should also give rise to intermediates in the nematocyte differentiation pathway, e.g., nests of four interstitial cells (4s; David and Gierer, 1974). Figure 5B shows that $4 \mathrm{~s}$ first appeared on Day 1 and increased exponentially with a doubling time (1.2 days) identical to that of the interstitial cell population. Since 4 s are differentiation intermediates, which are produced from stem cells and which turn into 8s (David and Gierer, 1974), the exponential increase in their numbers indicates that they are continuously produced during growth of the stem cell population.

\section{Peduncle Tissue Contains Stem Cells and Postmitotic Nerve Cell Precursors}

In summary, our results indicate that peduncle tissue contains two classes of interstitial cells: cells in G1 and cycling cells in S/G2. Essentially all G1 cells (70\% of total) are small interstitial cells; S/G2 cells (30\% of total) are a mixture of large and small interstitial cells. The G1 cells have the properties of postmitotic nerve cell precursors which have been previously described (Holstein and David, 1986). The S/G2 cells are cycling and, following transplantation to a gastric environment, support rapid exponential growth. Since these cells also give rise to nematocyte precursors, they are stem cells and not cycling nerve precursors. Although our results do not rigorously exclude low levels of cy- cling nerve precursors, the majority of cycling cells in peduncle tissue appear to be stem cells.

Since we only observe stem cells and postmitotic nerve cell precursors in peduncles, it appears reasonable to assume that stem cells are the immediate target of signals inducing nerve cell differentiation.

The authors thank Hans Bode for sharing unpublished results on peduncle interstitial cells in $H$. oligactis. We are very grateful to $B$. Hobmayer and Th. Bosch for critically reading the manuscript and to R. Kemeter and C. Griebe for their excellent technical assistance. This research was supported by the Deutsche Forschungsgemeinschaft (Da 163-1-2; Ho 1088-1-1).

\section{REFERENCES}

Bode, H., Berking, S., David, C. N., Gierer, A., Schaller, H., and TrEnkNer, E. (1973). Quantitative analysis of cell types during growth and regeneration in hydra. Wilhelm Roux' Arch. Entwicklungsmech. Org. 171, 269-285.

BoDE, H. R., and DAVID, C. N. (1978). Regulation of a multipotent stem cell, the interstitial cell of hydra. Prog. Biophys. Mol. Biol. 33, 189206.

BoDE, H. R., GEE, L. W., and CHOW, M. (1990). Neuron differentiation in hydra involves dividing intermediates. Dev. Biol. 139, 231-243.

DAvid, C. N. (1973). A quantitative method for maceration of hydra tissue. Wilhelm Roux' Arch. Entwicklungsmech. Org. 171, 259-268.

DAVID, C. N., and CAMPBELL, R. D. (1972). Cell cycle kinetics and development of Hydra attenuata. I. Epithelial cells. J. Cell Sci. 11, 557568.

DAvid, C. N., and Gierer, A. (1974). Cell cycle kinetics and development of Hydra attenuata. II. Nerve and nematocyte differentiation. J. Cell Sci. 16, 359-375.

David, C. N., Bosch, T. C. G., Hobmayer, B., Holstein, T., and SchmidT, T. (1987). Interstitial stem cells in hydra. In "Genetic Regulation of Development” (W. F. Loomis, Ed.), pp. 389-408. A. R. Liss, New York.

DiEHL, F. A., and BuRnETT, A. L. (1964). The role of interstitial cells in the maintenance of hydra. I. specific destruction of interstitial cells in normal, asexual and non-budding animals. J. Exp. Zool. 155, 253259.

HeIMFELd, S., and BoDE, H. R. (1984). Interstitial cell migration in Hydra attenuata. I. Selective migration of nerve cell precursors as the basis for position-dependent nerve cell differentiation. Dev. Biol. 105, 10-17.

HolsteIN, TH., and DAvid, C. N. (1986). The properties of nerve cell precursors in hydra. Dev. Biol. 115, 18-26.

Holstein, Th., Schaller, H. C., and David, C. N. (1986). Nerve cell differentiation in hydra requires two signals. Dev. Biol. 115, 9-17.

Holstein, TH. W., and David, C. N. (1990). Cell cycle length, cell size, and proliferation rate in hydra stem cells. Dev. Biol. 142, $392-400$.

RuBIN, D. I., and BoDE, H. R. (1982). The Aberrant, a morphological Mutant of Hydra attenuata, has altered inhibition properties. Dev. Biol. 89, 316-331.

SCHALLER, H. C. (1976). Action of head activator on the determination of interstitial cells in Hydra. Wilhelm Roux' Arch. Entwicklungsmech. Org. 180, 287-295.

Sugiyama, T., and FuJisawa, T. (1977). Genetic analysis of developmental mechanisms in hydra. I. Sexual reproduction of Hydra magnipapillata and isolation of mutants. Dev. Growth. Differ. 19, 187200 . 